\title{
Clinical, Hormonal and Metabolic Characterization of Mexican Women who had Gestational Diabetes in the Institute of Security and Social Services of workers of the State of Mexico (Issste)
}

\author{
JC Paredes Palma*1, Carlos Ramirez Velazquez ${ }^{2}$, Francisco Hernandez Fragoso ${ }^{3}$, Lorena \\ Balladares Macedo ${ }^{4}$ and Claudia N Paredes Palma ${ }^{5}$ \\ ${ }^{1}$ Gyneco Endocrinologist and Human Reproductive Biologist of the Hospital "Dr. Fernando Quiroz Guterrez" and Clinical Researcher of the ISSSTE, Mexico \\ ${ }^{2}$ Specialist in Allergy and Immunology of the Hospital “Dr. Fernando Quiroz Gutierrez" and Clinical Researcher of the ISSSTE, Mexico
}

${ }^{3}$ Head of Gynecology and Obestetrics Department of "Dr Dario Fernandez Fierro" Hospital of the Issste, Mexico

${ }^{4}$ Specialist in Gynecology and Obstetrics addressed to the Churubusco ISSSTE Clinic of Specialties, Mexico

${ }^{5}$ Specialist in Gynecology and Obstetrics and Clinical Researcher of the ISSSTE, Mexico

*Corresponding author: JC Paredes Palma, Gyneco Endocrinologist and Human Reproductive Biologist of the Hospital "Dr. Fernando Quiroz Guterrez" and Clinical Researcher of the ISSSTE, Mexico, Email: jcpp2024@hotmail.com, ju.paredes@issste.gob.mx

To Cite This Article: JC Paredes Palma. Clinical, Hormonal and Metabolic Characterization of Mexican Women who had Gestational Di abetes in the Institute of Security and Social Services of workers of the State of Mexico (Issste). Am J Biomed Sci \& Res. 2019 - 5(4). AJBSR. MS.ID.000927. DOI: 10.34297/AJBSR.2019.05.000927.

Received: 眥 September 18, 2019; Published: 眥 September 27, 2019

\begin{abstract}
Background: Diabetes Mellitus (DM2) has increased its incidence worldwide and in Mexico. The prevalence of Gestational Diabetes Mellitus in Mexico ranges from 4 to $14 \%$. The risk of postpartum transformation from DMG to DM2 is $40 \%$ and in other studies it is described as a 10 -fold risk. There is no work that has characterized clinically, metabolically and hormonally Mexican women who have suffered from GDM, to know the differences that exist with other described populations, establish ethnic risk factors and probable treatment strategies.
\end{abstract}

Material and Methods: A descriptive, cross-sectional study was conducted in 30 Mexican women who during 2018 and 2019 from 18 to 37 years old who were detected during pregnancy with DMG in a Second Level Care Hospital of the ISSSTE, clinical, metabolic characterization was performed and hormonal. Descriptive statistics, correlation of variables with Pearson's P were developed and Odds Ratio was determined for the development of Eclampsia and Preeclampsia.

Results: 30 women who suffered from Gestational Diabetes were detected, the age range fluctuated between 18 and 37 years with an average (P) of 28 years and standard deviation (SD) of 6.43. Most women detected had some degree of overweight or obesity, 26 patients (86.6\%). Insulin resistance detected in this population in terms of HOMA was established in 16 patients (53.3\%). In 5 (16.1\%) patients, fasting impaired glucose was determined and in $1(3.2 \%)$ Prediabetes due to glucose $>140$ at $2 \mathrm{hrs}$, as well as in 1 (3.2\%) Diabetes Mellitus. Most of the patients had some type of dyslipidemia 28 (93.3\%). The average of the group of patients in the case of Gestational Age at the end of pregnancy was 37.7 with a Standard deviation of 1.25 , with a range of 36 to 40 weeks gestation,

Conclusion: Most of the population of Mexican women who were studied for gestational diabetes had metabolic abnormalities more frequently than those reported in other populations with a similar prevalence of DM2 conversion reported in other ethnic groups.

Keywords: Mellitus Diabetes; Gestational Mellitus Diabetes; Mexican Women; Pregnancy; Insulin Resistance

\section{Background}

In the last 30 years the incidence of obesity has increased alarmingly and almost at the same time as the Metabolic Syndrome, which evolves to Type 2 Diabetes Mellitus (DM2) 1-6. In 2012, the International Diabetes Federation (IFD) estimated that more than 
371 million people lived with the disease and that 4.8 million people die from it worldwide [1,2].

The frequency of DM2 in Mexico, from 1998 to 2012, there has been a trend towards an increase of $4.7 \%$, from a morbidity rate of 342.1 to 358.2 cases per 100,000 inhabitants, specifically in 2012 , 418,797 patients were reported diagnosed with diabetes (which represents $0.4 \%$ of the Mexican population), $59 \%$ of the cases were female, with the age group 50-59 years old being the most affected, with a morbidity rate of $1,237.90$ cases per 100,000 inhabitants [25]. The prevalence of Gestational Diabetes Mellitus (GDM) varies according to geographic area and is closely linked to the human ethnic type [6]. It is reported that in regions such as the United States it is more frequent among the Latino, Hispanic, Native American population, African American and Asian. The prevalence is around $5 \%$ of the general population in regions such as Pakistan, Belgium, Denmark, Estonia, Ireland, South Korea, South Africa, United Kingdom and is around $10 \%$ of the general population for Italy, Turkey, Brazil, USA, and Australia. The prevalence is very high in regions such as Bermuda and Nepal with $20 \%$. In a recent report from the International Diabetes Federation, he estimates that $16 \%$ of births around the world in 2013 had complications from Gestational Diabetes 5, 6. With the consensus of the new criteria for DGs issued by both the American Association of Diabetes (ADA) as the European Society for the Study of Diabetes Mellitus the prevalence of the global DG will be around $18 \%$ by 2015 [1,5].

In Mexico, a frequency that varies between 4 and $14 \%$ has been described depending on literature [4]. The rate of cases that convert from DMG to DM 2 is still high (up to 40\%) and the effect of the treatments offered is not full. A risk of up to 7 times the risk of suffering from DM 2 was found in patients with a history of DMG [6].

Breastfeeding during the first 6 months of postpartum promotes beneficial effects on the product preventing overweight or obesity by $20 \%$ [7].

Gestational Diabetes Mellitus affects 14\% of pregnancies annually, and approximately $90 \%$ of cases of diabetes in pregnant women are considered as Gestational Diabetes Mellitus [1,5]. DMG is defined as the failure in glucose tolerance that begins during pregnancy. Many risk factors for developing DMG are similar for DM2, including obesity, family history for diabetes, high-risk ethnic group, other additional risk factors are increase in maternal age, history of birth of macrosomic products, as well as history of DMG in previous pregnancies [8].

The DMG appears mainly in women with easily identifiable risk factors such as: pregnancy changes that increase insulin resistance, glucose levels affect the development of the fetus and can cause macrosomia 8. Changes in Maternal physiology during pregnancy is due to the storage of energy in the fatty tissue that from the end of the second trimester has adjustments so that it is released and can be referred to the growing fetus $[3,7,9]$. Normal pregnancy is considered a stage Diabetogenic or progressive insulin resistance due to changes in the pattern of insulin secretion and changes in its sensitivity and its action [5].

This hormonal resistance of pregnant women seems to be due to a combination of maternal adiposity and desensitizing effects of various substances produced by the placenta (placental lactogen, placental growth hormone, prolactin, corticotropin-releasing hormone - cortisol, insulinases, tumor growth factor alpha, interleukin 6 , adiponectins (leptin, resistin and visfatin), which is evidenced by the rapid withdrawal of resistance almost 24 hours after delivery [3-5].

It is known that in normal pregnancy there are hormonal and metabolic changes mediated by placental hormones, in particular TNF- $\alpha$ and other factors related to previous obesity such as low molecular weight adiponectins and genetic predisposition, causing decreased insulin sensitivity, then the blood supply of insulin for available body glucose decreases by $50 \%$ so pregnant women have to increase their pancreatic secretion of insulin by $50 \%$ [10-12]. If this does not happen in this way, carbohydrate intolerance related to pregnancy or DMG occurs $[5,6]$.

In a study published in 2013 by Bonde L et al. a decrease in endogenous increment GLP-1 (glucagone like peptide-1) secretion was observed among women with DG at $2 \mathrm{hrs}$ after a standard load of 75 gr. of glucose, in the case of incretin GIP (glucagone like peptide) remained unchanged [13]. The latter corresponds to that observed by Lencioni C. et al. In 2011, it was observed that the secretion of GLP-1 is significantly lower in patients with DG compared to women with euglycemic pregnancy both during and after pregnancy, evidenced by a glucose tolerance curve with 100 grams of glucose [14].

There is a high risk of suffering from DM2 once you have suffered from DG at some time after delivery even when euglycemia is reached. In a Danish study it was found that $40 \%$ of women who suffered from DG treated with diet developed DM2 10 years after pregnancy $[1,6]$.

Compared to women between 30 and 60 years of age, women who suffered from DG have the risk of developing DG 10 times more.

In a systematic review of 20 studies, a risk of up to 7 times the risk of suffering from DM2 was found in patients who had DG compared to those with euglycemic pregnancy [1].

The progression of normal glucose tolerance (TNG) to diabetes is mediated by a transition state called glucose intolerance (IG) [11]. Important properties have been found for breastfeeding such as: facilitating the elimination of meconium, facilitating the production of lactobacilli in the intestine, immunoglobulins (lactoferrin, IgA, oligosaccharides, lymphocytes and macrophages) covering the inner lining of the digestive tract preventing adhesion of bacteria, viruses, parasites and thus avoiding allergic processes, growth factors stimulate the maturation of the child's own systems, such as 
the maturation of the digestive tract, its volume and osmolarity are appropriate for the kidney of the RN, nutritional superiority, immediate availability and Ideal temperature, immune support, promotes CNS maturation, and prevents malnutrition. Breastfeeding during the first 6 months of postpartum promote beneficial effects on the product preventing overweight or obesity by up to $20 \%$, however, the results are controversial, since strict adherence to breastfeeding and the time of breastfeeding are not specified this in the evaluation periods [12].

In the case of patients with normal blood glucose and who present IG, and in patients with impaired fasting glucose (GAA), insulin resistance and beta cell dysfunction are metabolic alterations that exist and share with patients who They have already developed Diabetes Mellitus 2 (DM2) and that trigger conversion [10-12].

In the initial stages of insulin resistance there is an increase in insulin secretion by beta cells, creating a hyperinsulinism state, however, the function of beta cells is impaired $[11,12]$.

In the case of the GAA, it also constitutes an intermediate metabolic condition between the TNG and the development of DM2, it is a different entity from the GI but together they coined the term "Prediabetes" accepted by the American Diabetes Society (ADA). The pathophysiology of both conditions has differences, as well as their impact on atherosclerosis [11].

In GAA, hepatic insulin resistance is increased and the initial insulin response in a glucose tolerance test is decreased (0-30 min). When hyperglycemic clamp techniques and intravenous glucose tolerance test are applied, the first phase of secretion is observed, but in the late phase 60 to $120 \mathrm{~min}$ the sensitivity to muscle insulin is practically normal and serum glucose returns to normal at 2 hrs In GI there is moderate to severe insulin muscle resistance and there is an alteration in insulin secretion in the early and late stages when a glucose tolerance test (CTG) is performed $[10,12]$.

The standard Gold for assessing insulin resistance is to calculate the insulin secretion/insulin resistance (disposition)/G IR index. Patients who have GI have 3 to 4 times the risk of developing DM2, in prospective epidemiological studies it has been observed that $40 \%$ of patients in follow-ups for 5 years develop DM2 when presenting GAA and IG without intervention [15].

Therefore, the strategies for changing the state of life and pharmacological treatment that impact these two conditions is rational to think that they will contribute to inhibit the progression of GI and GAA to DM2 [16].

To improve insulin resistance, a diet and aerobic program have been proposed as strategies, these strategies have shown to decrease the progression of GAA and IG to DM2 in 4 articles [15-19].

Both in the Finish Diabetes prevention program and the Lanmark DPP Study (dipeptidyl peptidase) it was observed that, when carrying out interventions in calorie intake, weight loss promotion and aerobic activity reduced the incidence of new cases in 58\% after a 2.8- and 3.2-year follow-up respectively [16,18].

However, the improvement in effective insulin sensitivity has not been satisfactory, which is why a series of pharmacological interventions have been carried out to improve it.

The use of metformin plus weight loss has been used in the Landmark DDP study and the Diabetes Program for Indians with a $31 \%$ decrease in the incidence of conversion to DM2, however, as in the case of modifications of the lifestyle does not break the damage in the beta cell and therefore the effective progression to DM2 $[16,18]$.

The thiazolidediones act as activator of the peroxisome proliferation range receptors (PPAR-y) as well as intervene in the two metabolic alterations observed in patients with IG and GAA, namely insulin resistance and pancreatic beta cell dysfunction. There have been no statistically significant differences in the improvement in insulin resistance and pancreatic beta cell functioning between pioglitazone, troglitazone, rosiglitazone. In the case of troglitazone, a similar effect was observed in glycemic control and insulin sensitivity compared to the other thiazolidinediones mentioned and it has been observed that the incidence of IG conversion and patients suffering from Gestational Diabetes (DG) has been reduced by $23 \%$ and was more efficient when compared against placebo, metformin or lifestyle changes. In the DREAM study, a decrease in the incidence of DM2 from IG to DM2 attributed to rosiglitazone was observed in $62 \%$ and improvement in beta function through the measurement of insulin secretion / insulin resistance index. Pioglitazone and troglitazone have shown a decrease in the incidence of DM2 in patients with IG who had a history of DG [16-19].

In the ACTOS Now study, a decrease in the incidence of DM2 was observed in patients with IG of $72 \%$ attributed to pioglitazon. 27 The inconvenience with the management of thiazolidinediones while improving the metabolic alterations shared by IG and GAA and triggering DM2 conversion is water retention, weight gain / fat. However, in a study conducted in Canadians, rosiglitazone $2 \mathrm{mg}$ daily plus $1000 \mathrm{mg}$ daily of metformin was used in patients with IG found a decrease in conversion to DM2 by $71 \%$, without statistically significant differences in weight gain and water retention [20].

Analog incretins which is one of the innovative treatments for patients with DM2 and which together have been called GLP-1 analogs (glucagone like peptide-1).

Oral glucose consumption produces an increase in insulin secretion 3 to 4 times higher compared to intravenous glucose instillation, this is called "incretin effect", and is 95\% mediated by GIP secretion (glucagone like peptide) and GLP-1 secreted through L and intestinal cells respectively [21].

GLP-1 also inhibits gastric emptying, has an anorexigenic effect, produces gradual weight loss, decreases endothelial oxidative 
stress, and in vitro studies the decrease in apoptosis of pancreatic beta cells has been observed [21]

A study conducted with GLP-1 followed for 3 years showed in patients with DM2, the sustained reduction of glycosylated hemoglobin (Hb-g) as well as weight loss and improvement of beta function. GLP-1 does not produce hypoglycemia since insulin secretion is based on the presence of hyperglycemia [22].

In a clinical trial it was observed that GLP-1 after one treatment continued for 3 years produced an improvement in the response of beta cells to glucose [22].

The lack of adequate prevention of the conversion of patients with GAA and IG is due to the damage in the beta cell that so far, no pharmacological treatment has performed in total. GLP-1 increases the response of beta cells to glucose, promoting improvement in their functioning, and also inhibits their early apoptosis evidenced in patients with diabetes, produces weight loss and produces an effect at the level of peripheral insulin resistance and are highly safe since the frequency of hypoglycemia is practically nil As a group of medications, they could be candidates to evaluate their effectiveness in preventing the conversion of IG and GAA to DM2. Within this group of medications, Liraglutide has been studied in non-diabetic obese patients where the incidence of conversion of GAA and IG to DM2 was reduced from 84 to $96 \%$, and they lost weight by $5 \%$ in $61 \%$ of patients. patients and a $10 \%$ reduction in weight was obtained in $19 \%$ of individuals [22-24].

According to the differences in the pharmacokinetics and pharmacodynamics of GLP1, Liraglutide is considered the analogue with better effects on the other GLP-1.

There are no studies available to evaluate its effectiveness in prediabetes states. A very important limitation in the groups of medications used for prediabetes states is the lack of sustainability of the inhibitory effect of the conversion of impaired fasting glucose and impaired glucose (GAA) to DM2 after stopping the use of medication despite to continue with an adequate lifestyle. A new group of drugs called GLP 1 analogues showed that their effect is favorable on glycosylated hemoglobin, with a decrease in body weight maintained over time and with a low risk of hypoglycemia, a favorable metabolic profile on lipids and also pressure adequate systolic blood pressure in diabetic patients 25 and its use in prediabetic obese patients decreased the conversion to DM 2 by $84 \%$ to $96 \%$ [24-26]. Liraglutide at a dose of $1.8 \mathrm{mg}$ daily subcutaneously was the most effective dose in studies conducted to control obesity.25 and therefore, long-acting GLP-1 could be drugs of choice in order to avoid conversion of IGT to DM type 2, because it modifies the pathophysiology of the disease, with which the progressive deterioration of pancreatic beta cells in addition to decreased body weight maintained over time and with a low risk of hypoglycemia, with a favorable metabolic profile on lipids and systolic blood pressure [21-24].
In the Mexican population there are no data to establish the clinical, metabolic and hormonal characteristics of patients who suffered from Gestational Diabetes in order to determine if the behavior is comparable to that reported in other populations.

\section{Overall Objective}

Characterize a population of women from a second level of care hospital of the ISSSTE, who suffered Gestational Diabetes in the metabolic, hormonal and anthropometric aspects.

\section{Specific Objectives}

1. Determine alterations in glucose metabolism in women who suffered from gestational diabetes Based on a $5 \mathrm{hrs}$. Glucose Tolerance Curve with 75 grams of glucose

2. Determine insulin secretion in women who suffered from gestational diabetes based on a 5 hrs. Glucose Tolerance Curve with 75 grams of glucose.

3. Determine the degree of insulin resistance in women who suffered from gestational diabetes based on the calculation of the HOMA index

4. Determine lipid metabolisms in women who suffered gestational diabetes based on the quantification of their lipid profile.

5. Determine thyroid, pituitary and adrenal functioning in women who suffered from gestational diabetes based on the quantification of their pituitary and thyroid profile.

6. Characterize the anthropometric profile in women who suffered from gestational diabetes

\section{Material and Methods}

A cross-sectional study was conducted in 30 patients from the "Dr. General Hospital. Darío Fernandez Fierro" of the ISSSTE who suffered from gestational diabetes, detected in the first 6 months after birth, over 18 years of age during the second half of 2018 and first half of 2019, according to the criteria of Hyperglycemia and Adverse Pregnancy Outcomes (HAPO).

The hormonal and metabolic quantification was performed with chemiluminescence and Elisa technique with coefficients of variation intra and inter analysis $<10 \%$

Descriptive statistics and Pearson's P tests were performed for bivariate correlation.

\section{Results}

During the last semester of 2018 and first semester of 2019, 30 women who suffered from Gestational Diabetes were detected, the age range fluctuated between 18 and 37 years with an average (P) of 28 years and standard deviation (SD) of 6.43 (Figure 1).

Most of the women detected had some degree of overweight or obesity, 26 patients (86.6\%), 11 were overweight (36.6\%), 8 wom- 
en were obese grade I (26.6\%), 8 were obese grade II $(26.6 \%), 2$ $(6.4 \%)$ patients had grade III obesity and 5 morbid obesity (16.6\%) (Figure 2).

Insulin resistance detected in this population in terms of HOMA was established in 16 patients (53.3\%). In 5 (16.1\%) patients, fast- ing impaired glucose was determined and in 1 (3.2\%) Prediabetes due to glucose $>140$ at $2 \mathrm{hrs}$, as well as in 1 (3.2\%) Diabetes Mellitus. Hypoglycemia occurred in the first part of the glucose tolerance curve in 7 patients and in the final part of the curve in 10 patients (33.33\%) (Figure 3).

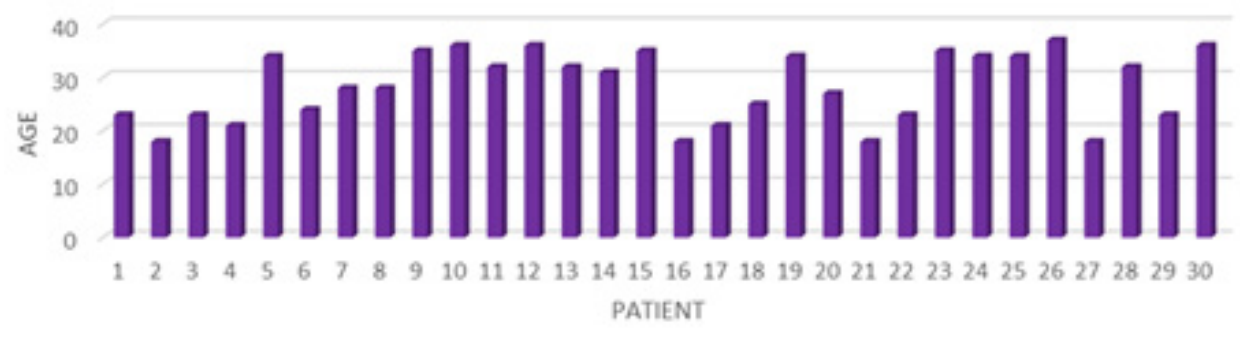

Figure 1: Distribution by age of Mexican women who were Gestational Diabetes.

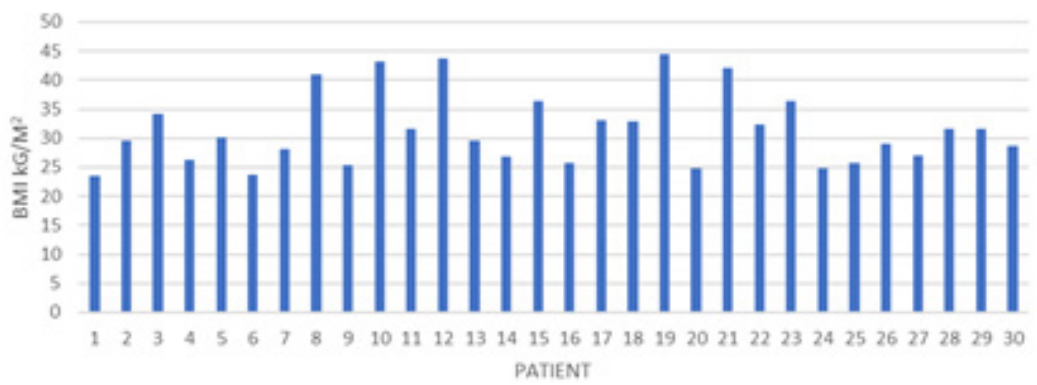

Figure 2: Body Mass Index After the Delivery in Mexican women who had Gestational Diabetes.

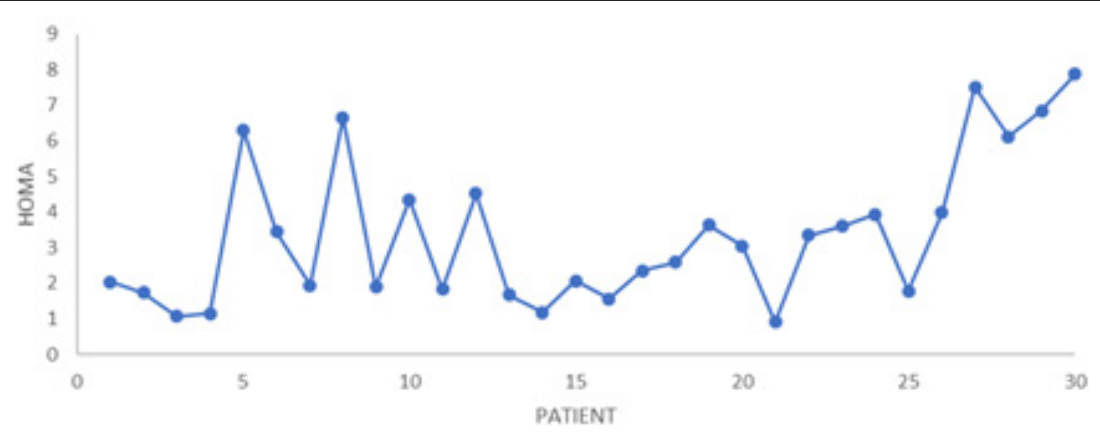

Figure 3: Insulin Resistance in Mexican Women After the Delivery who had Gestational Diabetes.

In relation to dysinsulinism (hyperinsulinism) in the first part had the alteration at the end of the curve of integrated insulin seof insulin secretion after a stimulation of 75 grams of glucose 29 cretion (Figure 4,5).

(93.54\%) patients presented said alteration and 15 (50\%) patients

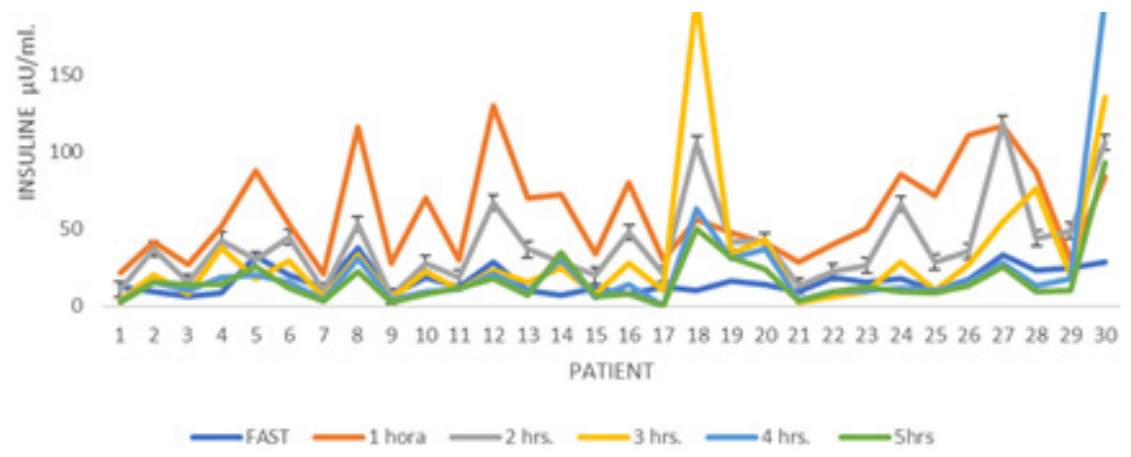

Figure 4: Insulin Secretion curve of Mexican Women After the Delivery who had Gestational Diabetes with 75 Grs of glucose. 


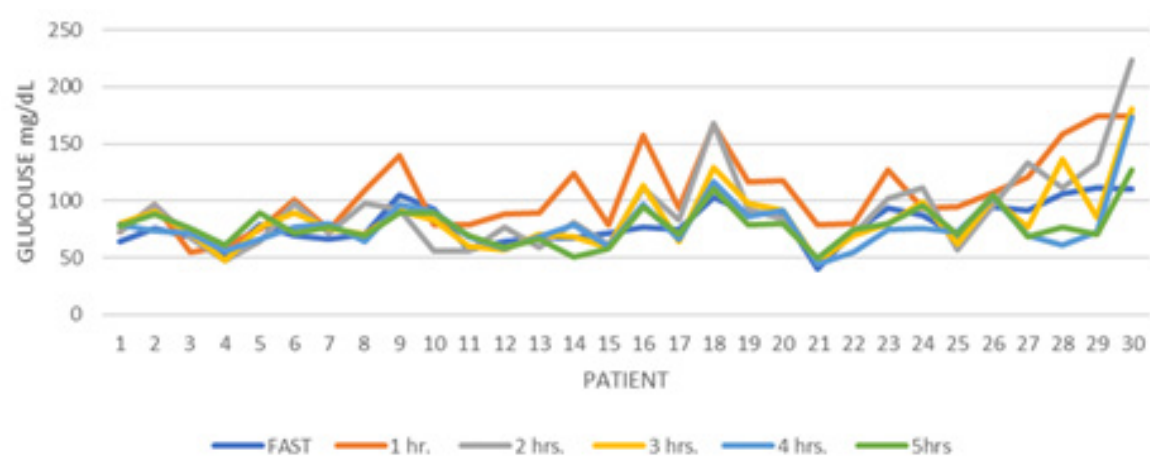

Figure 5: Glucose Secretion curve of Mexican Women After the Delivery who had Gestational Diabetes with 75 Grs of glucose.

A glycosylated $\mathrm{Hb}$ in the ranges of Diabetes Mellitus was pre- ria for the diagnosis of Diabetes mellitus (Figure 6). sented in 6 women, however in 5 patients there are no other crite-

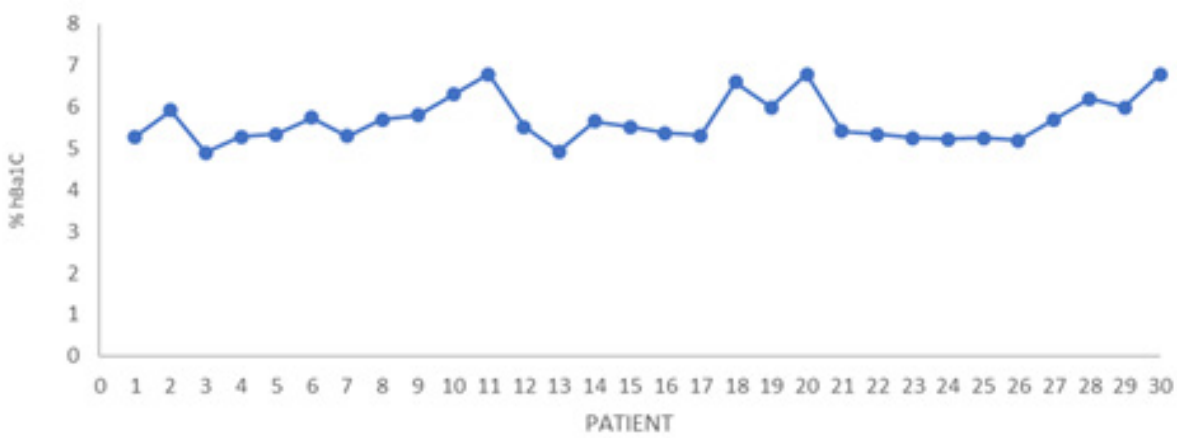

Figure 6: Glucose Secretion curve of Mexican Women After the Delivery who had Gestational Diabetes with 75 Grs of glucose.

Most of the patients had some type of dyslipidemia 28 (93.3\%),

24 (80\%) women presented hypercholesterolemia, 8 (26.6\%) women hypertriglyceridemia, 8 (26.6\%) apolipoproterinemia and 20 (66.6\%) presented LDL-hypercholesterolemia (Figure 7).
In terms of thyroid functioning, 8 patients had some type of dysfunction, $6(20 \%)$ of them presented criteria for primary hypothyroidism and 2 (6.6\%) for secondary hypothyroidism.

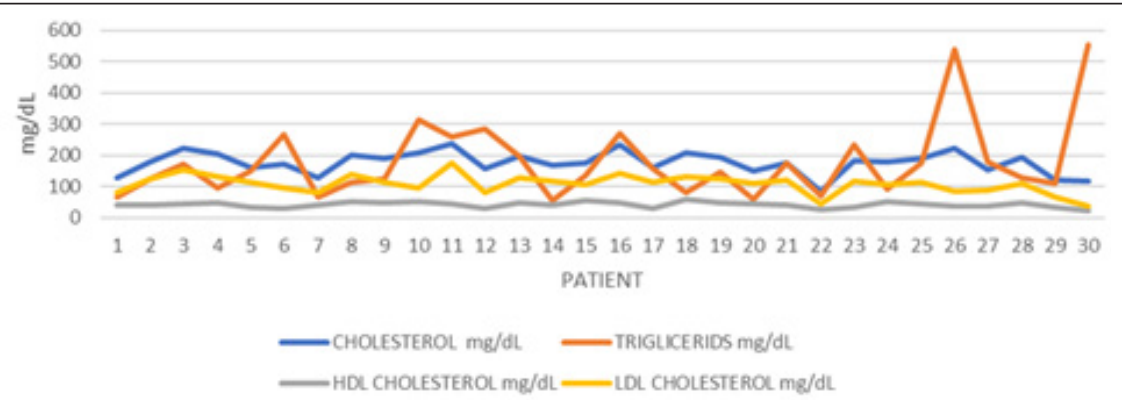

Figure 7: Lipid profile after the Delivery in Mexican women who had Gestational Diabetes.

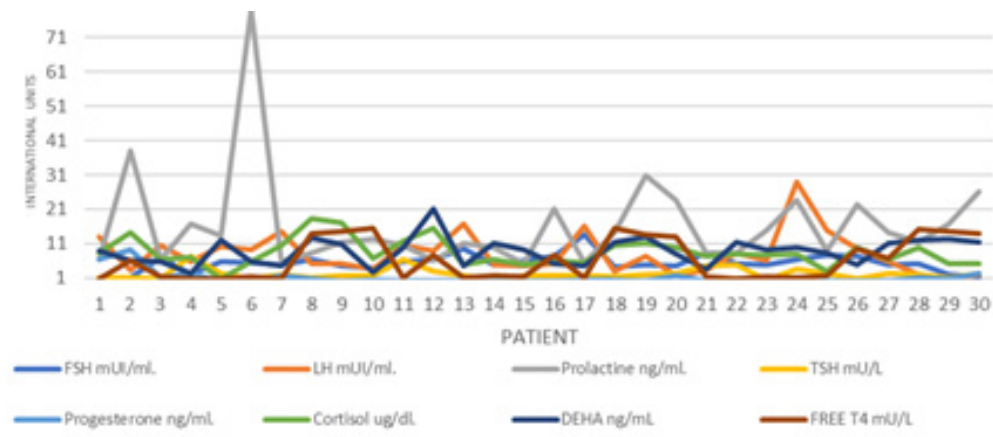

Figure 8: Hypofisary profile after the Delivery in Mexican women who had Gestational Diabetes. 
In the pituitary hormonal profile, the Stimulating Follicle Hormone (FSH) was observed in 5 (16.6\%) women in abnormal ranges in the follicular phase, suggesting a decrease in ovarian reserve, as well as $5(16.6 \%)$ patients with hypoestrogenism despite having a Normal FSH. All patients had values in normal ranges of Corticotropic Hormone (ACTH), however morning hypocortisolism occurred in $2(6.6 \%)$ patients (Figure 8$)$.

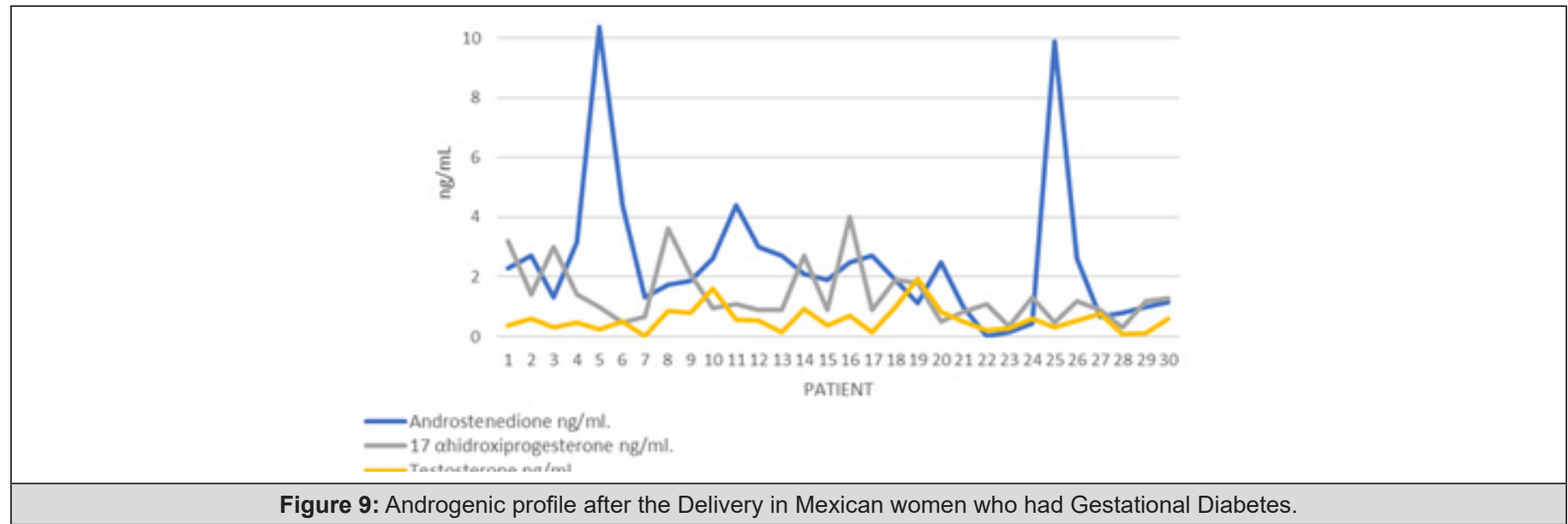

Figure 9: Androgenic profile after the Delivery in Mexican women who had Gestational Diabetes.

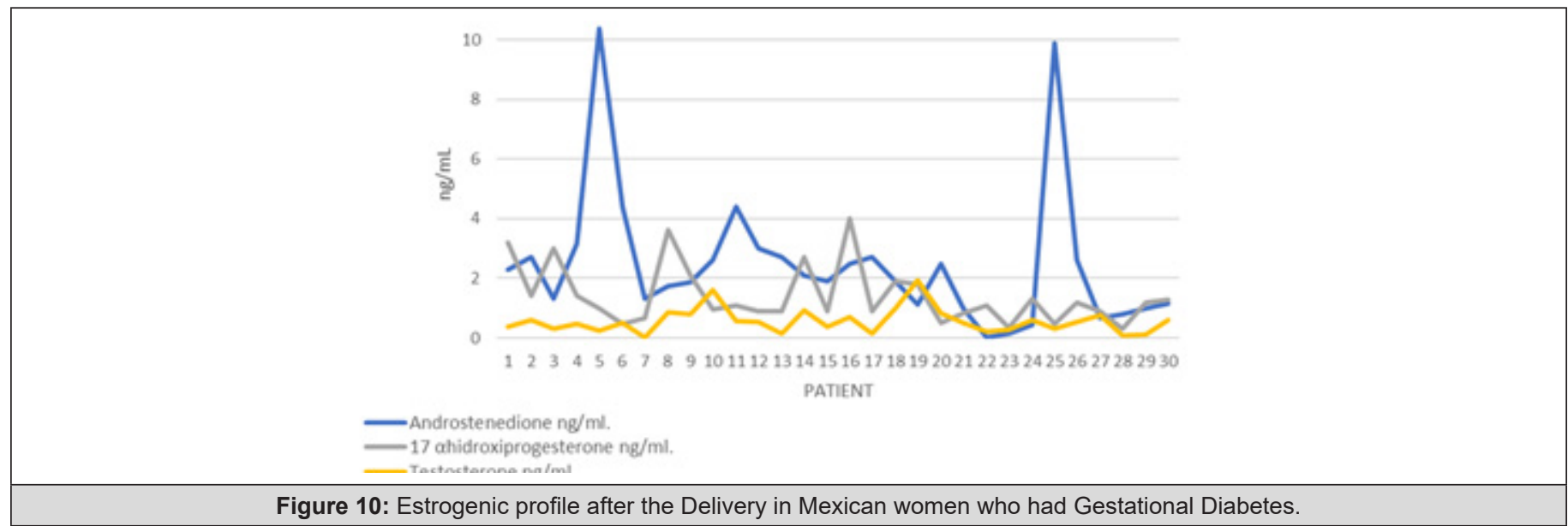

In 4 patients, hyperandrogenism related to increased testosterone and androstenedione was found, 1 in tumor range for testosterone. 3 patients presented abnormally high ranges of 17 hydroxyprogesterone (170HP4) in ranges compatible with polycystic ovary (Figure 9,10)
The average of the group of patients in the case of Gestational Age at the end of pregnancy was 37.7 with a Standard deviation of 1.25 , with a range of 36 to 40 weeks of gestation, in 7 (23.3\%) patients presented as a preclamsia complication and only in an eclampsia and in no Help Syndrome (Figure 11).

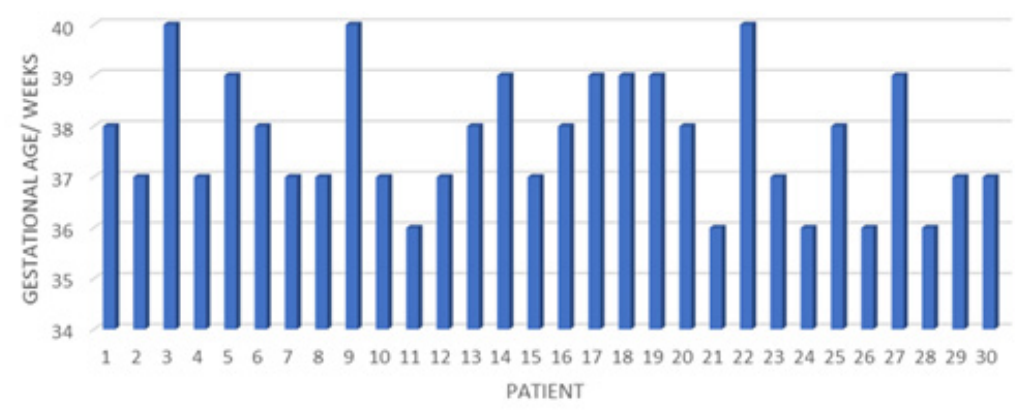

Figure 11: Gestational age at Birth in Women who had Gestational Diabetes.

Regarding the bivariate analysis, a positive correlation was found between insulin resistance in terms of HOMA Vs Gestational Age (P Pearson 0.14) and HOMA Vs age (P Pearson 0.2) as well as a significantly positive correlation between HOMA Vs Triglycerides and $\mathrm{Hb}$ glycosylated (P Pearson 0.30 ) and significantly negative among HOMA Vs Total Cholesterol, LDL and HDL. (P Pearson -0.30). A positive correlation of HOMA Vs. Estradiol (P Pearson 0.22) was observed (Figure 12,13). 


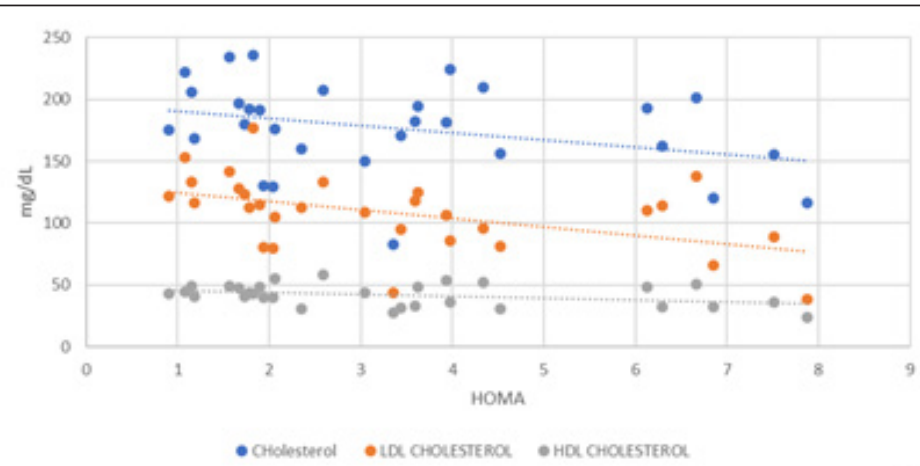

Figure 12: Correlation Between Total Cholesterol and LDL, HDL Cholesterol Vs Homa in Women who had Gestational Diabetes after Delivery.

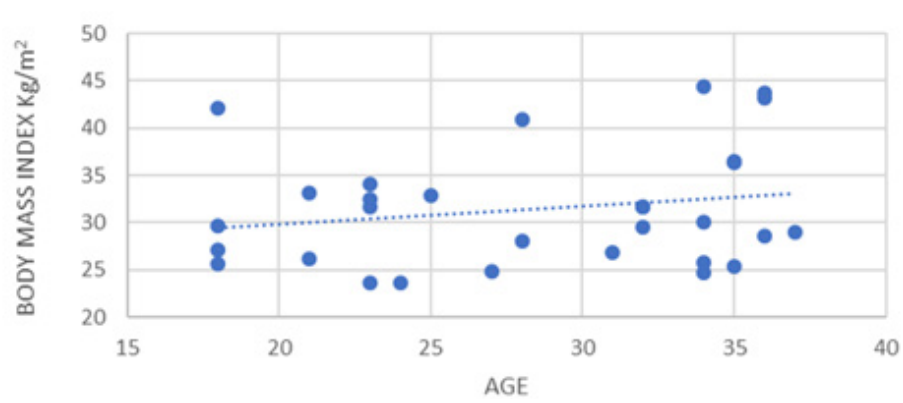

Figure 13: Correlation Between Index of Body Mass and Age in Women who had Gestational Diabetes after Delivery.

With respect to BMI, a significantly positive correlation was Pearson 0.20) (Figure 14). observed with baseline insulin (P Pearson 0.23) and with age (P

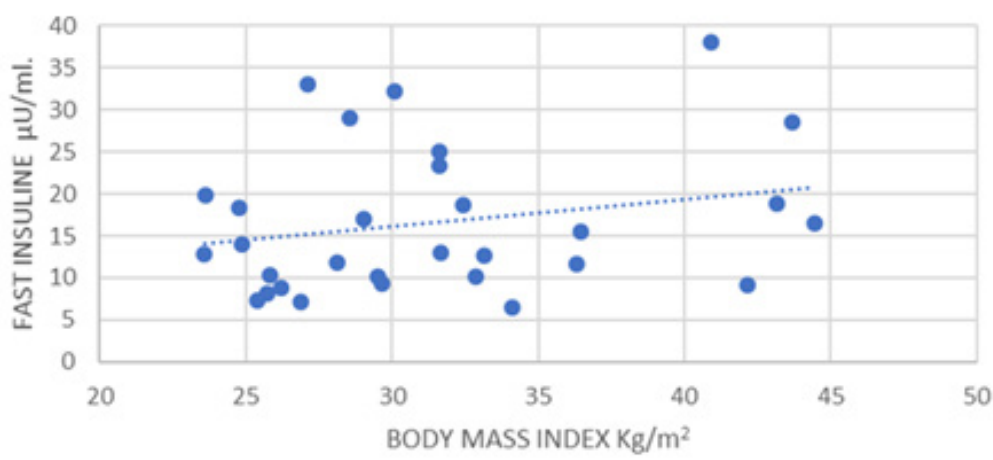

Figure 14: Correlation Between Index of Body Mass and Fasting Insulin in Women who had Gestational Diabetes after Delivery.

It is pertinent to consider that a negative correlation was ob- relation was observed between basal insulin Vs Cholesterol, LDL served between BMI, Basal Insulin Vs Gestational Age (P Pearson and HDL (P Pearson -0.31, -0.37 and -0.39 respectively) (Figure 15). -0.14 and -0.15 respectively). Similarly, a significantly negative cor-

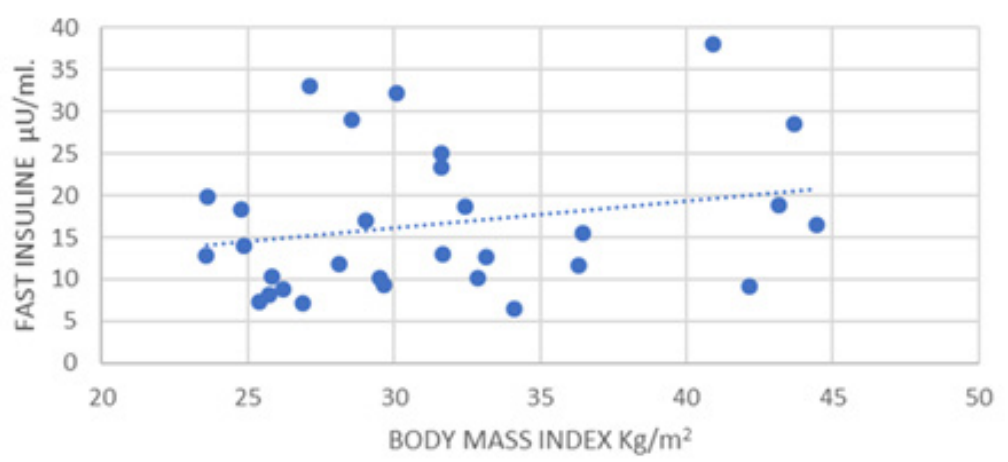

Figure 15: Correlation Between Total Cholesterol and LDL, HDL Cholesterol and Fasting Insulin in Women who had Gestational Diabetes after Delivery. 
In the case of the Odds Ratio (OR) calculated with respect to insulin resistance (HOMA), dysinsulinism, impaired fasting glucose and the risk of suffering from Preclamsia or Eclampsia, no statistical significance was obtained in the calculation of risk.

\section{Discussion}

The results include that the majority of patients (80\%) who suffered from gestational diabetes, when performing their clinical and metabolic evaluation, presented some type of alteration in glucose metabolism, insulin secretion and lipid metabolism (53\% Resistance to Insulin, $16.1 \%$ impaired fasting glucose, $3.2 \%$ carbohydrate intolerance and 3.2\% were detected with DM2 that jeopardize their health and the imminent development of DM2, results comparable to those observed by Katrien Benhalima et al. [25]. in 2016 where 135 American women who studied after childbirth after having attended pregnancy with Gestational Diabetes, $42.2 \%$ developed Prediabetes (11.9\% impaired fasting glucose and $24.4 \%$ carbohydrate intolerance and 59\% presented both alterations) and in $1.5 \%$ they detected Diabetes Mellitus in an Italian study led by Brunetti et al. [26]. In 2014, observed the prevalence of prediabetes (impaired glucose fasting and carbohydrate intolerance per curve) in $32.1 \%$ postpartum in a cohort of 234 patients and DM2 of $4 \%$ postpartum. Chew Wf et al. [27]. In 2012, I studied 448 Malaysian women who had Gestational Diabetes, finding a prevalence of Postpartum Prediabetes at $26.2 \%$ and $35.5 \%$ for DM2.

Among the risk factors that can condition these alterations observed in the results it should be noted that $86.6 \%$ presented some degree of overweight or obesity (11 overweight women (36.6\%), 8 women obesity grade I (26.6\%), 8 obesity degree II (26.6\%), 2 (6.4\%) grade III obesity and 5 morbid obesity (16.6\%), a similar trend was observed in the population studied by Katrien Benhalima et al. [25]. Where 33.8\% of some degree of overweight was observed or obesity in the study population In the case of the weeks of gestation where the birth occurred, in our study it was an average of $37.7 \pm 1.25$ a week with a range of 36 to 40 weeks of gestation and in the population studied by Katrien Benhalima et al. [25] it was $38.1 \pm 2.2$.

In the case of the study of Brunetti ET AL [26]. It was observed as the main predictive factor for the development of postpartum prediabetes, the previous diagnosis of Polycystic Ovarian Syndrome and for the development of postpartum DM ", the Body Mass Index> 25 pre pregnancy as well as a fasting glucose $>100$ at the time of diagnosis of Gestational Diabetes Mellitus. In the case of our work, the tendency as risk factors for the development of complications of pregnancy (preclamsia and eclampsia) to impaired fasting glucose, dysinsulinism and insulin resistance, although they did not reach statistical significance most likely due to the size of the sample.

\section{Conclusion}

Most of the population of Mexican women who were studied for gestational diabetes had metabolic abnormalities more frequently than those reported in other populations with a similar prevalence of DM2 conversion reported in other ethnic groups.

\section{Reference}

1. Ferrara A (2007) Increasing prevalence of gestational diabetes mellitus. A public health perspective. Diabetes Care 30(2): S141-S146.

2. Juan López Mercedes Secretaria de Salud (2013) Pablo Antonio Kuri Morales Subsecretario de prevención y promoción de la salud. Boletín epidemiológico de Diabetes Mellitus tipo 2, primer trimestre p. 1-25.

3. García García Carlos (2008) Diabetes Mellitus Gestacional. Medicina Interna de México 24(2): 148-156.

4. Tamez H, Rodríguez M, Treviño M, Espinosa J, et al. (1993) Experiencia con un programa de escrutinio de diabetes gestacional. Rev Invest Clin 45: 453-456.

5. Gutiérrez HI, Carrillo M, Pestaña S, Santamaría M (2006) Pacientes diabéticas embarazadas: experiencia institucional. Ginecol Obstet Mex 74(4): 187-192.

6. Mustafa Kanat, Ralph A Defronzo, Muhammad A Abdul (2015) Treatment of Prediabetes. World Journal of Diabetes 6(12): 1207-1222.

7. Simone Y, Chang Ye, Ravi Retnakaran (2011) Postpartum Metabolic Function in Women Delivering a Macrosomic Infant in the Absence of Gestational Diabetes Mellitus. Diabetes care 34(12): 2608-2613.

8. Ravi Retnakaran, Chang Ye Anthony J G, Hanley et al. (2012) Effect of maternal weight, adipokines, glucose intolerance and lipid on infant birth weight among women without Gestational Diabetes Mellitus. CMAJ 184(12): 1353-1360

9. Desoye G, Hauguel-de Mouzon S (2007) The human placenta in gestational diabetes mellitus. The insulin and cytokine network. Diabetes Care 30(2): S120-S126.

10. Winter C, Wagner O, Festa A, Schneider B, Roden M, et al. (2004) Plasma adiponectin, insulin sensitivity, and subclinical inflammation in women with prior gestational diabetes mellitus. Diabetes Care 27(7): 17211727.

11. Shireen Brewster, John Floras, Bernard Zinman, Ravi Retnakaran (2013) Endothelial function in women with and without a history of glucose intolerance in pregnancy. J Diabetes Research 2: 1-9.

12. Ravi Retnakaran, Ying Qi, Philip W Connelly, Mathew Sermer, et al. (2010) Glucose intolerance in Pregnancy and Postpartum risk of Metabolic Syndrome in young women. J Clin Endocrinol Metab 95(2): 670677.

13. Bonde L, Vislsboll T, Nielsen T, Bagger Jl, Svare JA, et al. (2013) Reduce postprandial GLP-1 response in women with gestational diabetes mellitus. Diabetes Obes Metab 15(8): 713-720.

14. Lencioni C, Ressi V, Romero F, Lupi R Volpe L, Bertolloto A, et al. (2011) Glucagon -Like peptide-1 secretion in women with gestational diabetes mellitus during and after pregnancy. J Endocrinol Invest 34(9): 287-290.

15. Tieu J, Middleton P, McPhee AJ, Crowther CA (2014) Screening and subsequent management for gestational diabetes for improving maternal and infant health. Cochrane Database Syst Rev 11(2): CD007222.

16. Merlotti C, Morabito A, Pontiroli AE (2014) Prevention of type 2 diabetes; a systematic review and meta-analysis of different intervention strategies. Diabetes Obest Metab 16(8): 719-727.

17. Turner RC, Cull CA, Frighi V, Holman RR (1999) Glycemic control with diet, sulfonylurea, metformin, or insulin in patients with type 2 diabetes mellitus: progressive requirement for multiple therapies (UKPDS 49). UK Prospective Diabetes Study (UKPDS) Group. JAMA 281(21): 20052012.

18. Kelley KW, Carroll DG, Meyer A (2015) A review of current treatment strategies for gestational diabetes mellitus. Drug context 15(4): 212282.

19. (2015) American Diabetes Association. Management of Diabetes in pregnancy. Diabetes Care 38(1): s77-s79. 
20. (2009) Study protocol Open Access Actos Now for the prevention of diabetes (ACT NOW) study BMC Endocrine Disorders 9: 17.

21. Holst JJ (2006) Glucagon-like peptide-1: from extract to agent. The Claude Bernard Lecture, 2005. Diabetologia 49(2): 253-260.

22. Madsbad S (2009) Liraglutide effect and action in diabetes (LEAD $\left.{ }^{\mathrm{TM}}\right)$ trial. Expert Rev Endocrinol Metab 4(2): 119-129.

23. Astrup A, Rössner S, Van Gaal L, Rissanen A, Niskanen L, et al. (2009) Effects of liraglutide in the treatment of obesity: a randomized, double-blind, placebo-controlled study. Lancet 374(9701): 1606-1616.

24. Nauck M, Frid A, Hermansen K, Shah NS, Tankova T, et al. (2009) Efficacy and safety comparison of liraglutide, glimepiride, and placebo, all in combination with metformin, in type 2 diabetes: the LEAD (liraglutide effect and action in diabetes)-2 study. Diabetes Care 32: 84-90.
25. Katrien Benhalima, Katleen Jegers, Roland Devlieger, Johan Verhaeghe, Chantal Mathieu (2016) Glucose Intolerance after a Recent History of Gestational Diabetes Based on the 2013 WHO Criteria. PLoS One 11(6): e0157272.

26. Capula C, Chiefari E, Vero A, Foti DP, Brunetti A, et al. (2014) Prevalence and predictors of postpartum glucose intolerance in Italian women with gestational diabetes mellitus. Diabetes Res Clin Pract 105(2): 223-230.

27. Chew WF, Rokiah P, Chan SP, Chee WS, Lee LF, et al. (2012) Prevalence of glucose intolerance and associated antenatal and historical risk factors among Malaysian women with a history of gestational diabetes mellitus. Singapore Med J 53(12): 814-820. 